\title{
To the Problem of Neuro circulating Saevs
}

\author{
Shaposhnikov Veniamin Ivanovich* \\ Department of Morphological Disciplines, Professor of The Department of Surgical Diseases, Vice Rector for Science of the \\ Noncommercial educational private institution of higher education "Kuban Medical Institute, Russia
}

*Corresponding author: Shaposhnikov Veniamin Ivanovich, D.M.D., Professor, Department of Morphological Disciplines, Professor of The Department of Surgical Diseases, Vice Rector for Science of the Noncommercial educational private

institution of higher education "Kuban Medical Institute, Russia

\section{ARTICLE INFO}

Received: 幽 March 06, 2020

Published: 蔧 March 17, 2020

Citation: Shaposhnikov Veniamin Ivanovich. To the Problem of Neuro circulating Saevs. Biomed J Sci \& Tech Res 26(4)-2020. BJSTR. MS.ID.004383.

\section{ABSTRACT}

A simple way of reflex therapy in vegetative dystonia is described. It provides for the effect on the parasympathetic nerves of the brain stem through active points located on the skin of the face, a blunt dense object with a diameter of $1.0-1.5 \mathrm{~mm}$. This eliminates skin injury in the con-tact area and makes it completely bloodless. It is devoid of all the negative properties inherent in drug therapy, does not require special technical equipment and other material costs, and can at any time be easily and effectively performed by the patient suffering from this disease. For all these reasons, it needs to be widely publicized among the population.

Keywords: Vegetative-Vascular Dystonia; Parasympathetic Nerves of the Head; Sparing Mechanical Reflexology

\section{The Aim of the Study}

Develop a public gentle way of correcting pathological symptoms in vegetative dystonia through the use of mechanical reflexology.

\section{Short Communication}

If consciousness depends on the function of the cerebral cortex, the senses are associated with the activ-ity of the autonomic nervous system, which is divided into two divisions - sympathetic (strengthens the heart rhythm, narrows the vessels, oppresses peristaltic gastrointestinal tract, etc.) and parasympathetic (expands blood vessels, slows down heart contractions, strengthens bowel motor skills, etc.). Thus, the first of them activates the energy costs of the body, and the second restores them. Usually this system functions as a whole without causing any painful sensations [1-3]. When this equilibrium is disturbed, then immediately there is a pathological symptom complex, which usually prevails pathology associated with the sympathetic department - there is tachycardia, spasm of blood vessels, headache and heart ache, intestinal colic and so on $[4,5]$. In old age, especially at night, it can lead to cardiac arrest and breathing. In the devel-opment of this pathological pro cess can take part, as mediators of pain (serotonin, bradicinin and acetylcho-line) - contributing to the production of adrenaline, and endorphins (hormones of bliss) - depressing and even blocking the respiratory center $[6,7]$.

In a child and a teenager, the main cause of these pathological processes is related to the hereditary factor. Up to $70 \%$ of them suffer from headache, $45 \%$ - experience increased fatigue during physical exertion, 30\% - dizziness and nausea when the body is changed. When a person grows up, a social factor is added. This creates reasons for the development of a variety of functional disorders, which were previously described as "vegetative-vascular dystonia" and now, as neuroses, encephalopathy, and neurocirculatory disorders $[4,8]$. In the development of this disease highlight the following causal factors:

1) Psychogenic trauma with emotional stresses,

2) Excessive physical activity with a violation of the regime of work, rest, nutrition and healthy lifestyle,

3) Sexual incontinence or incontinence or Conversely impotence with endocrine disorders, 
4) Disruption of the function of internal organs, bone and joint and other systems, causing pronounced discomfort and feelings of uncertainty in society, etc.

Depending on the nature of the pathological reaction, they occur:

1) By cardiac type,

2) By hypotonic type,

3) By hypertensive type,

4) By mixed type,

5) By vagotonic type,

6) By somatic (neurotic) type.

First, functional disorders occur in the vegetative nervous system, and then they turn into persistent chronic diseases, with the most frequent suffering cardiovascular and respiratory systems. In this case, the diagnosis of the body's functional reserves is determined by assessing cardio-respiratory synchronism. Involvement in the patho-logical process [8,9]. Involvement in the pathological process of the digestive tract is accompanied by flatulence and stool disorders (constipation, diarrhea). In the absence of adequate treatment develop neuroses, depression, panic attacks, migraine. The person becomes irritable and suffers from chronic fatigue. The resulting imbalance between the sympathetic and parasympathetic departments of the vegetative system leads to a violation of tissue perfusion and hormone secretion. Other vital mechanisms of homeostasis stability suffer. There is a real threat to the patient's life.

In this situation, the normalization of the parasympathetic department becomes vital. Usually the person copes with this problem - during sleep or physical rest. During sleep, nutrients from the intestines are increased into the internal environment of the body, unoxidized metabolic products are disposed of, hormones and trace elements are distributed to organs and tissues, as well as other vital ones are made processes. It is important that they be carried out regularly and unhindered. If this does not happen, then treatment is required. Currently, the list of drugs used in this disease has significantly expanded - teturam, zorex, esperal, methadhoxyl, vazalamine, etc. $[5,10,11]$. All the authors emphasize the importance of leading a healthy lifestyle, eating right (taking more fish, fruits and vegetables), exercising, outdoor activities and sleep patterns, while avoiding stress, anxiety, inferiority complex. It should be remembered that this disease is reversible if a person is able to eliminate the effects on the body of adverse factors in time. Thus, at present, the drug with which you can achieve recovery from this disease, and sometimes medicines on the contrary cause other serious diseases (e.g., chronic pancreatitis).
Moreover, sometimes it is necessary to immediately stabilize the function of the cardiovascular system, and the tablet does not have time to show its medicinal properties. For this reason, the use of non-traditional medicine in the treatment algorithm for this disease has a certain positive effect, as they allow to immediately remove the patient from a critical condition, and then continue treatment without threat development of allergies and other related chronic diseases caused by the patient's sensitivity to medication. This method deserves special attention in pregnancy, if it is accompanied by an allergy of the mother, as the patient with the help of reflex therapy sometimes saves not only herself, but also her fetus. However, it is necessary not only to own it, but also to have technical equipment - or needles, or magnetic field, or laser, or device, regenerating electrical impulse, which makes the independent execution of this procedure is not real. However, a simpler method of doing it is possible. It does not require any technical equipment or medical knowledge on the part of the person who will, or himself, or his friend, provide this medical care. It is effective, safe and quite simple, and therefore is generally available. This article is dedicated to him.

\section{Materials and Methods}

During 30 years of teaching at the medical school, it was necessary to constantly observe vegetative dystonia in senior students, especially during the session. They resorted to the pill, which could lead to negative consequences. It seemed more appropriate to use their reflexology by direct mechanical effect on the skin endings on the face $1,5,7,10$ parasympathetic nerves of the brain stem. As you know, they carry out the perception of sensory impulses, as well as partially involved in respiratory, digestive and cardiac activities. Such anatomical structure allows a person to actively influence his homeostasis that is to regulate and activate the reserve capabilities of his body, if to produce irritation on the face ending these nerves. Thus, a person can actively influence the preservation of the vitality of the body. The most important for reflex therapy active points are located: in the area of skin contact of the upper lip with the nasal septum, in the pits near the right and left wing of the nose, on the chin, on the nose, at the lower poles of the pineal growths. For massage it is convenient to use any narrow long dense object with a diameter of 1.0 -1.5 mm. Contact circular mechanical impact, before the appearance of tolerable pain, you should start from the upper lip, and then move from one area to another.

Manipulation should continue until the disappearance of developed pathological syndrome. It usually takes 3-5 minutes. I personally described reflexology, was carried out many times. Most of them were students between the ages of 20 and 24 . They all had a mixed form of vegetative dystonia. The procedure was carried out in the presence of the whole group and was both therapeutic and informative. It was important to teach all students how to perform 
this type of reflex therapy on their own, so that they could then successfully perform it if the need arises. This goal was achieved in $100 \%$ observations. They did not need to have a second-in-face procedure. About mastering the technique of its conduct, they themselves told in the next classes. This method was also used in patients with any surgical chronic disease occurring against the background of vegetative dystonia. Approximately 30-50 patients were treated. Each time there was a positive effect, but repeated manipulations were required, as patients showed a striking indifference to their fate, that is, were lazy to carry out this procedure, considering it a matter of medical staff. In this situation, it was simply impossible to expect a permanent correction of the disease.

\section{Results}

All patients have a positive effect. No complications were observed. When exposed to active zones, patients experienced tolerable pain and sometimes numbness of the skin. It was espe-cially common in the upper lip area. After the procedure, their pulse slowed down and im-mediately becomes satisfactory filling and tension. Patients' condition improved markedly. There was no need to repeat the procedure, but sometimes it was repeated 2-3 times due to scientific and medical reasons.

\section{Discussion}

The described method of reflex therapy at a young age gives a persistent positive result. It al-lows the patient to actively participate in the restoration of the vital tone of the body and to correct its functional reserves. He has confidence in his studies, in sports, in the arts and in other prestigious events. He is not afraid of physical and emotional stress, because he knows that if necessary, he can always easily and simply help himself, without resorting to medicines that can be expensive. Moreover, it can always help parents, rela- tives and acquaintances, which increases its importance in society and all this easily and easily and without any side-effects.

\section{Conclusion}

Mechanical point reflexology on the face without skin damage can be attributed to promising ways of treating neurocirculatory disorders (vegetative dystonia), as it allows a person personally easy and simple, and most importantly effective to correct homeostasis and stimulate the body's functional reserves.

\section{References}

1. Bryn VB (2016) Normal Physiology. Edited by BI Tkachenko. (3 $3^{\text {rd }}$ Edn). And dop M GEOTAR - Media, pp. 688s.

2. Degtyarev VP, Sorokina ND (2016) Normal Physiology. Textbook. GEOTAR - Media, pp. 480.

3. (2012) J West Breathing Physiology. Basics. LitTerra, 312c.

4. Kamkin AG, Kiseleva IS (2013) Atlas in Two Volumes. M GEOTAR - Media, pp. 408s.

5. Kogan UA, Kruglikov GG, Celuiko SS (2013) Respiratory Pathology. Atlas. Editor VS Paukov. Lit Terra Publishing House, pp. 530.

6. Kurzanov AN, Zabolotsky NV, Manuilov AM (2015) Clinical and physiological aspects of the diagnosis of functional reserves of the body. Cuban Scientific Medical Gazette 6: 73-77.

7. Kuselman AI (2006) Endorphins function, excess and hormone deficiency. Methodical recommendations. M GEOTAR - Media, pp. 288.

8. Pokrovskiy VM (2010) Heart-breathing synchronism in the assessment of the body's regulatory and adaptive capabilities. Kuban-book, Krasnodar, Russia, pp. 244.

9. Teal LS, Aghajanyan NA (2015) Normal Physiology. Textbook. M Lit Terra, pp. 768.

10. Tkachenko NT (2003) To the issue of the treatment of panic attacks. Cuban Scientific Medical Gazette 1-2: 155-156.

11. Fedorova VI (2000) Vegetative disorders in persons with asthenic syndrome and their correction of malat citrullin /Neurology and Psychiatry 4: 34-36.

\section{ISSN: 2574-1241}

\section{DOI: $10.26717 /$ BJSTR.2020.26.004383}

Shaposhnikov Veniamin Ivanovich. Biomed J Sci \& Tech Res

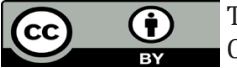

This work is licensed under Creative Commons Attribution 4.0 License

Submission Link: https://biomedres.us/submit-manuscript.php

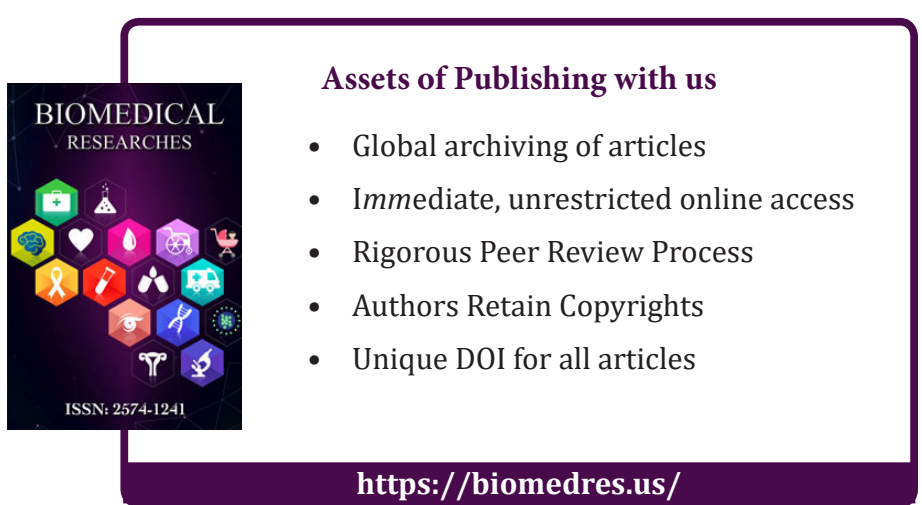

